It is not well established that long-term contact to low concentration of toluene produces changes in male hormonal profile (MHP).

Objective To identify changes in the MHP, consisting of luteinizing hormone $(\mathrm{LH})$, follicle-stimulating hormone (FSH) and testosterone, in workers exposed to toluene in an industrial packaging plant in Mexico City.

Material and Methods Cross-sectional study that included 42 workers, from which were formed two groups: with high (HET) and low (LET) exposure to toluene; serum FSH, LH, testosterone and acid hippuric in urine were measured in all subjects.

RESULTS Hippuric acid in subjects with LET: $2.53 \pm 1.20 \mathrm{~g} / \mathrm{g}$ creatinine, and with HET: $6.31 \pm 3.83 \mathrm{~g} / \mathrm{g}$ creatinine $(\mathrm{p}=$ 0.02). Seric FSH concentration: $5.12 \pm 0.77$ and $3.55 \pm 0.3$ $\mathrm{mU} / \mathrm{mL}(\mathrm{p}=0.02)$ in LET and HET respectively; LH: $2.66 \pm$ 0.45 and $2.77 \pm 0.21(\mathrm{p}=0.81)$, and testosterone: $3.91 \pm$ 0.34 and $4.86 \pm 0.23 \mathrm{ng} / \mathrm{mL}(\mathrm{p}=0.04)$. By regression analysis, the correlation coefficient of FSH with hippuric acid: $-0,182(\mathrm{p}$ $=0.031$, with coefficient of determination of $11 \%$, the LH: $0,007(\mathrm{p}=0.88)$ and $0.05 \%$ respectively, and testosterone: + $0209(\mathrm{p}=0.0001)$ and $34 \%$.

Conclusions The effect of toluene is evident on FSH; $\mathrm{LH}$ also decreased but not overwhelming; testosterone seems to have opposite response, perhaps explained by different sensitivity of the male gonads to toluene exposure. These findings appear to be the initial changes in MHP of workers exposed to the solvent in question.

\section{EXPOSURE TO POLYCYCLIC AROMATIC HYDROCARBONS AND SPERM DNA INTEGRITY OF COKE OVEN WORKERS}

${ }^{1} \mathrm{C}$ Jeng, ${ }^{2} \mathrm{Pan},{ }^{3}$ Lin. ${ }^{1}$ Old Dominion University, Norfolk, United States of America; ${ }^{2}$ Institute of Occupational Safety and Health, Taipei, Taiwan; ${ }^{3}$ Kaohsiung Municipal Hsiaokang Hospital, Kaohsiung, Taiwan

\subsection{6/oemed-2013-101717.95}

The objective of this study was to examine sperm DNA integrity of coke-oven workers in relation to exposure to PAHs from coke processing. A total of 72 human subjects participated in this study: 24 topside-oven workers, 28 side-oven workers, and 20 administrative personnel serving as the high exposure group, low exposure group, and control, respectively. An exposure assessment was conducted to depict the extent of $\mathrm{PAH}$ exposure by measuring urinary 1-hydroxypyrene (1OHP). DNA fragmentation, 8-oxo-7,8-dihydro-2'-deoxyguanosine (8-oxodGuo), and bulky DNA adducts in sperm DNA were quantified using terminal deoxynucleotidyl transferasemediated dUTP nick end labelling, liquid chromatographymass spectrometry/mass spectrometry, and 32P-labelling, respectively. Coke-oven workers had increased levels of DNA fragmentation, 8-oxodGuo, and bulky DNA adducts. However, there was no significant difference in DNA fragmentation levels among the three studied groups $(P=0.062)$. The levels of 8 -oxodGuo and bulky DNA adducts in the exposed groups were significantly higher than those in the control $(P=0.048$ and 0.032 , respectively), while controlling for age, alcohol consumption, and smoking. DNA fragmentation positively correlated with 8-oxodGuo, which suggests that oxidative stress may be linked to DNA breakage. Urinary 1-OHP levels did correlate with 8 -oxodGuo levels $(P=0.036)$, but not bulky DNA adducts and DNA fragmentation. In summary, exposure to PAHs correlated with oxidative damage and formation of DNA adducts in sperm. Monitoring of sperm DNA integrity is recommended for affected workers as part of any periodic health assessment to determine the impact of occupational toxins on sperm.

\section{MENSTRUAL CYCLE CHARACTERISTICS IN EUROPEAN AND INUIT WOMEN EXPOSED TO PERFLUORINATED CHEMICALS: PRELIMINARY FINDINGS FROM A CROSS- SECTIONAL STUDY}

${ }^{1}$ L J Lyngsoe, ${ }^{2}$ Ramlau-Hansen, ${ }^{3}$ Høyer, ${ }^{4}$ Bonde, ${ }^{3}$ Toft. ${ }^{1}$ Aarhus C, Denmark; ${ }^{2}$ Aarhus University, Aarhus C, Denmark; ${ }^{3}$ Aarhus University Hospital, Aarhus C, Denmark; ${ }^{4}$ Copenhagen University Hospital Bispebjerg, Copenhagen, Denmark

\subsection{6/oemed-2013-101717.96}

Objectives Perfluorooctanate (PFOA) and perfluorooctane sulfonate (PFOS) are ubiquitous man-made compounds. Studies suggest that they are possible hormonal disruptors, but findings are inconsistent. We examined the association between measured PFOS and PFOA exposure and menstrual cycle length and irregularities in European and Inuit women.

Methods This cross-sectional analysis included 1,037 pregnant women from the INUENDO cohort, enrolled during antenatal care visits between June 2002 and May2004 in Greenland, Poland and Ukraine. Information on menstrual cycle characteristics were obtained by questionnaires and the woman had a blood sample drawn. Serum concentrations of PFOS and PFOA were measured by liquid chromatography tandem mass spectrometry (LC/MS/MS). The association between PFOS/PFOA and menstrual cycle length and irregularities were analysed using logistic regression with tertiles of exposure and stratified by country. Estimates are given as crude odds ratios (ORs) with 95\% confidence intervals (CIs).

Results No consistent effects of PFOS and PFOA exposure on menstrual cycle characteristics were observed across all three groups of pregnant women. Within populations, we observed reduced odds of short cycles ( $\leq 24$ days) among women from Ukraine exposed to high levels of PFOA (OR 0.38, 95\% CI 0.15-0.97). However, in Greenland representing the highest exposure level, PFOA was not related to short cycles (OR 1.06, 95\% CI 0.21-5.34).

Conclusions These preliminary findings on 1,037 pregnant women from the INUENDO cohort in Greenland, Poland and Ukraine suggest that it is unlikely that exposure to PFOA and PFOS is a main cause of menstrual disturbances.

\section{FACTORS ASSOCIATED WITH RETURN TO WORK 6 MONTHS AFTER DELIVERY}

S P Pongpramorn. Srinakharinwirot University, Bangkok, Thailand

\subsection{6/oemed-2013-101717.97}

Nowadays, there is increasing non-return to work in pregnant workers. Therefore, the objective of this study was to explore the association between general characteristics, occupational, maternal and infant factors and women returning to work after pregnancy for further improve occupational health service in this population. The retrospective cohort study was conducted in July 2012 at Nopparat Rajthanee Hospital, Bangkok. The data were interviewing workers at Well baby clinic, Nopparat Rajthanee Hospital and Satellite Health Centers. The total population was women who delivered at this hospital in January 2012 and 